\title{
Article
}

\section{Fractional frequency Laplace transform by inverse difference operator with shift value}

\author{
Sandra Pinelas ${ }^{1, *}$, Meganathan Murugesan ${ }^{2}$ and Britto Antony Xavier Gnanaprakasam ${ }^{2}$ \\ 1 Departamento de Ciências Exactas e Naturais. Av. Conde Castro Guimarães, 2720-113 Amadora, Portugal. \\ 2 Department of Mathematics, Sacred Heart College, Tirupattur - 635601, Vellore District Tamil Nadu, South India.; \\ meganathanmath@gmail.com(M.M); brittoshc@gmail.com(G.B.A.X)) \\ * Correspondence: sandra.pinelas@gmail.com
}

Received: 5 November 2018; Accepted: 12 March 2019; Published: 29 April 2019.

\begin{abstract}
In this paper, we study the outcome of fractional Laplace transform using inverse difference operator with shift value. By the definition of convolution product, the properties of fractional transformation, the relation between convolution product and fractional frequency Laplace transform with shift value have been discussed. Further, the connection between usual Laplace transform and fractional frequency Laplace transform with shift value are also presented. Numerical examples with graphs are verified and generated by MATLAB.
\end{abstract}

Keywords: Fractional Laplace transform, polynomial factorials, exponential function, convolution product, inverse difference operator, trigonometric function, shift value.

MSC: 42A85, 49A10, 26D05, 39A70, 26A33, 46F12.

\section{Introduction}

$\mathbf{T}$ he continuous fractional calculus has been developed by Miller and Ross [1], Oldham and Spanier [2], and Podlubny [3]. Recently discrete delta fractional calculus have been developed by Atici and Eloe [4-6], Goodrich [7-9], and Holm [10]. These theories are used in integral transforms in the literature and are applied in astronomy, physics and engineering. The integral transforms like mellin, Laplace and Fourier were applied to obtain the solution of differential equations. These transforms made effectively possible changes a signal in the time domain into a frequency s-domain in the field of Digital Signal Processing(DSP) [11].

The delta Laplace transform was first defined in a very general way by Bohner and Peterson [12]. In 2015, Aleksandar Ivic discussed the discrete Laplace transforms in the view of fast decay factor $e^{-s x}$ and obtained the Laplace transform of $P(x)$ as $\int_{0}^{\infty} P(x) e^{-s x} d x=\pi s^{-2} \sum_{n=1}^{\infty} r(n) e^{-\pi^{2} / n}$. In practice, many applications of Laplace Transform (LT), $L[f(x)]=\int_{0}^{\infty} f(x) e^{-s x} d x$, and the forward Discrete Laplace Transform (DLT), $L[f(n)]=\sum_{n=0}^{\infty} f(n) e^{-s n}$, are discussed and mentioned by several authors in the citations [13-16].

In the existing Laplace transform the shifting value of time domains are one. In 2016, G. Britto Antony Xavier etc., [17] have defined Laplace transform with shift value $\ell$ using generalized difference operator and obtain the outcomes of polynomial and trigonometric functions etc. In this fractional Laplace transform with shift value $v$ taken from 0 to 1 .

In this paper, we continue the work derived in [17] by defining fractional frequency Laplace transform with fractional factor $e^{-s^{1 / v} t}$. We presented convolution product and several properties of the fractional transforms for the functions like polynomial factorial and trigonometric functions.

\section{Preliminaries}

In this section, we present basic theory of the $\ell$-difference operator $\Delta_{h}$. The polynomial factorial is defined $t_{h}^{(m)}=t(t-h)(t-2 h) \cdots(t-(m-1) h), h>0$ for non-negative integer $m$ and using Stirling numbers 
of first kind $s_{r}^{m}$ and second kind $S_{r}^{m}$, the relation between polynomial and polynomial factorials are given by,

$$
\text { (i) } t_{h}^{(m)}=\sum_{r=1}^{m} s_{r}^{m} h^{m-r} t^{r}, \quad \text { (ii) } t^{m}=\sum_{r=1}^{m} S_{r}^{m} h^{m-r} t_{h}^{(r)}
$$

Definition 1. Let $u(t), t \in[0, \infty)$, be a real or complex valued function and $h>0$ be a fixed shift value. Then, the $h$-difference operator $\Delta_{h}$ on $u(t)$ is defined as

$$
\Delta_{h} u(t)=\frac{u(t+h)-u(t)}{h}
$$

and its infinite $h$ - difference sum is defined by

$$
\Delta_{h}^{-1} u(t)=h \sum_{r=0}^{\infty} u(t+r h)
$$

Definition 2. Let $u(t)$ and $v(t)$ are the two real valued functions defined on $(-\infty, \infty)$ and if $\Delta_{h} v(t)=u(t)$, then the finite inverse principle law is given by

$$
v(t)-v(t-m h)=h \sum_{r=1}^{m} u(t-r h), m \in Z^{+}
$$

Applying the Definition 1, we get the modified identities as follows:

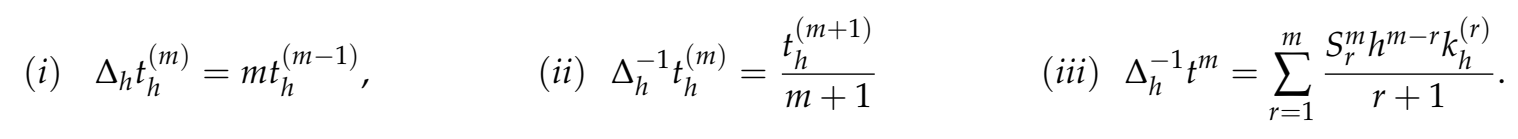

Lemma 3. [18] Let $h>0$ and $u(t), w(t)$ are real valued bounded functions. Then

$$
\Delta_{h}^{-1}(u(t) w(t))=u(t) \Delta_{h}^{-1} w(t)-\Delta_{h}^{-1}\left(\Delta_{h}^{-1} w(t+h) \Delta_{h} u(t)\right) .
$$

Lemma 4. Let $t \in(-\infty, \infty), h>0$ and $v>0$, then we have

$$
\Delta_{h}^{-1} e^{-s^{1 / v} t}=\frac{h e^{-s^{1 / v} t}}{\left(e^{-s^{1 / v} h}-1\right)}
$$

Proof. The proof follows by taking $u(t)=e^{-s^{1 / v} t}$ in Definition 1 and applying $\Delta_{h}^{-1}$.

Corollary 5. Let $t \in(-\infty, \infty), h>0$ and $v>0$, then we have

$$
\frac{h e^{-s^{1 / v} t}}{\left(e^{-s^{1 / v} h}-1\right)}-\frac{h e^{-s^{1 / v}(t-m h)}}{\left(e^{-s^{1 / v} h}-1\right)}=h \sum_{r=1}^{m} u(t-r h) .
$$

Proof. The proof follows by equating (7) and the finite inverse principle law given in (4).

Example 1. For the particular values $v=0.5, s=0.1, t=3$ and $h=2,(8)$ is verified by MATLAB. The coding is given by $(2 . * \exp (-(0.1) . \wedge(1 . / 0.5) . * 3)) . /(\exp (-(0.1) . \wedge(1 . / 0.5) . * 2)-1)-(2 . * \exp (-(0.1) . \wedge(1 . / 0.5) . *$ $1)) . /(\exp (-(0.1) . \wedge(1 . / 0.5) . * 2)-1)=2 . * \operatorname{symsum}(\exp (-(0.1) . \wedge(1 . / 0.5) . *(3-2 . * r)), r, 1,1)$.

Theorem 6. Let $t \in(-\infty, \infty), h>0$ be shift value and $2\left(\cosh s^{1 / v} h-\cos p h\right) \neq 0$. Then we have

$$
\begin{aligned}
& \Delta_{h}^{-1}\left(e^{-s^{1 / v} t} \cos p t\right)=\frac{h e^{-s^{1 / v} t}\left(e^{-s^{1 / v} h} \cos p(t-h)\right)-\cos p t}{2\left(\cosh s^{1 / v} h-\cos p h\right)}, \\
& \Delta_{h}^{-1}\left(e^{-s^{1 / v} t} \sin p t\right)=\frac{h e^{-s^{1 / v} t}\left(e^{-s^{1 / v} h} \sin p(t-h)\right)-\sin p t}{2\left(\cosh s^{1 / v} h-\cos p h\right)} .
\end{aligned}
$$


Proof. Taking $\Delta_{h}^{-1}$ on $u(t)=e^{-s^{1 / v} t} \cos p t$, we get:

$$
\Delta_{h}^{-1}\left(e^{-s^{1 / v} t} \cos p t\right)=\operatorname{Re} \text { part } \Delta_{h}^{-1}\left(e^{-s^{1 / v} t} e^{i p t}\right)=\operatorname{Re} \operatorname{part} \Delta_{h}^{-1}\left(e^{\left(-s^{1 / v}+i p\right) t}\right),
$$

Now (9) follows by applying Lemma 4 and taking conjugate. Similarly the proof of (10) holds.

\section{Fractional Frequency Laplace Transform and its Properties}

In this section, we define and obtain the properties of FGLT and present the transforms of certain functions like trigonometric, hyperbolic and polynomials etc.

Definition 7. Let $u(t)$ be the real valued function, $h>0$ and $v \in R^{+}$. If $\lim _{t \rightarrow \infty} \Delta_{h}^{-1} u(t) e^{-s^{1 / v} t}=0$, then the Fractional Frequency Laplace Transform(FFLT) is defined as

$$
L_{h, v}[u(t)]=\bar{u}_{h, v}(s)=\left.\Delta_{h}^{-1} u(t) e^{-s^{1 / v} t}\right|_{0} ^{\infty}=h \sum_{r=0}^{\infty} u(r h) e^{-s^{1 / v} r h} .
$$

Proposition 8. If $L_{h, v}(u(t))=\bar{u}_{h, v}(s)$ and $L_{h, v}(v(t))=\bar{v}_{h, v}(s)$, then

$$
L_{h, v}(a u(t)+b v(t))=a \bar{u}_{h, v}(s)+b \bar{v}_{h, v}(s) \quad \text { and } \quad L_{h, v}(u(a t))=\frac{1}{a} \bar{u}_{h, v}\left(\frac{s}{a}\right) .
$$

Proof. From (11), we have $L_{h, v}(u(a t))=\left.\Delta_{h}^{-1} u(a t) e^{-s^{1 / v} t}\right|_{t=0} ^{\infty}$. Now the proof follows by substituting at by $k$.

Proposition 9. If $L_{h, v}(u(t))=\bar{u}_{h, v}(s)$, then $L_{h, v}\left(e^{-a t} u(t)\right)=\bar{u}_{h, v}(s+a)$.

Proof. The proof follows by taking $u(t)=e^{-a t} u(t)$ in (11).

Theorem 10. If $\cosh s^{1 / v} h-\cos p h \neq 0$, then we have

$$
L_{h, v}[\sin p t]=\frac{h e^{-s^{1 / v} h} \sin p h}{2\left(\cosh s^{1 / v} h-\cos p h\right)} \text { and } L_{h, v}[\cos p t]=\frac{h\left(1-e^{-s^{1 / v} h} \cos p h\right)}{2\left(\cosh s^{1 / v} h-\cos p h\right)} .
$$

When $h \rightarrow 0$ and $v=1$, we get $L(\sin p t)=\frac{p}{s^{2}+p^{2}}$ and $L(\cos p t)=\frac{s}{s^{2}+p^{2}}$.

Proof. The proof of (13) follows from (9), (10) and (11).

Theorem 11. If $\cosh s^{1 / v} h-\cos (n-2 r) p h \neq 0$ for $r=0,1,2, \cdots, n$, then

$$
\begin{gathered}
L_{h, v}\left(\sin ^{n} p t\right)=\sum_{r=0}^{[n / 2]}\left(\begin{array}{l}
n \\
r
\end{array}\right) \frac{(-1)^{\left(\frac{n-1}{2}\right)+r} h \sin (n-2 r) p h}{2^{n}\left(\cosh s^{1 / v} h-\cos (n-2 r) p h\right)}, \quad n \text { is odd. } \\
L_{h, v}\left(\sin ^{n} p t\right)=\sum_{r=0}^{[n / 2]-1}\left(\begin{array}{l}
n \\
r
\end{array}\right) \frac{(-1)^{\frac{n}{2}+r} h\left(e^{s^{1 / v} h}-\cos (n-2 r) p h\right)}{2^{n}\left(\cosh s^{1 / v} h-\cos (n-2 r) p h\right)}+\left(\begin{array}{l}
n \\
\frac{n}{2}
\end{array}\right) \frac{(-1)^{\frac{n}{2} 2^{-n} h}}{\left(1-e^{-s^{1 / v}}\right)}, n \text { is even. } \\
L_{h, v}\left(\cos ^{n} p t\right)=\sum_{r=0}^{[n / 2]}\left(\begin{array}{l}
n \\
r
\end{array}\right) \frac{h\left(e^{s^{1 / v} h}-\cos (n-2 r) p h\right)}{2^{n}\left(\cosh s^{1 / v} h-\cos (n-2 r) p h\right)}, \quad n \text { is odd. } \\
L_{h, v}\left(\cos ^{n} p t\right)=\sum_{r=0}^{[n / 2]-1}\left(\begin{array}{l}
n \\
r
\end{array}\right) \frac{2^{-n} h\left(e^{s^{1 / v} h}-\cos (n-2 r) p h\right)}{\left(\cosh s^{1 / v} h-\cos (n-2 r) p h\right)}+\left(\begin{array}{l}
n \\
\frac{n}{2}
\end{array}\right) \frac{2^{-n} h}{\left(1-e^{-s^{1 / v} h}\right)}, n \text { is even. }
\end{gathered}
$$

Proof. From $\sin ^{n} p t=\frac{1}{2^{n-1}(-1)^{\frac{n-1}{2}}} \sum_{r=0}^{[n / 2]}(-1)^{r}\left(\begin{array}{c}n \\ r\end{array}\right) \sin (n-2 r) p t$ and (13), we get the proof of (14). Similarly we can obtain the proof of (15), (16) and (17). 
Corollary 12. Let $t \in[0, \infty), s, h, n u>0$, then we have

$$
h \sum_{r=0}^{\infty} u(r h) e^{-s^{1 / v} r h}=\frac{h \sin 5 p h}{32\left(\cosh s^{1 / v} h-\cos 5 p h\right)}-\frac{5 h \sin 3 p h}{32\left(\cosh s^{1 / v} h-\cos 3 p h\right)}+\frac{10 h \sin 5 p h}{32\left(\cosh s^{1 / v} h-\cos p h\right)} .
$$

Proof. The proof follows by taking $n=5$ in (14) and then equating that with (11).

Example 2. For the particular values $v=0.6, s=3, p=2$ and $h=3,(18)$ is verified by MATLAB. The coding is given by $3 . * \operatorname{symsum}((\sin (2 . * 3 . * r)) . \wedge 5 . * \exp (-3 . \wedge(1 . / 0.6) * * 3 . * r), r, 0$, inf $)=(3 . * \sin (5 . * 2 . *$ $3)) . /(32 . *(\cosh ((3) . \wedge(1 . / 0.6) . * 3)-\cos (5 . * 2 . * 3)))-(5 . * 3 . * \sin (3 . * 2 . * 3)) . /(32 . *(\cosh ((3) . \wedge(1 . / 0.6) . *$ $3)-\cos (3 . * 2 \cdot * 3)))+(10 * * 3 . * \sin (2 . * 3)) . /(32 . *(\cosh ((3) . \wedge(1 . / 0.6) \cdot * 3)-\cos (2 . * 3)))$.

Theorem 13. If $e^{-\left(s^{1 / v} \pm p\right) h} \neq 1$ and $s>0$, then

$$
L_{h, v}(\sinh p t)=\frac{h}{2}\left(\frac{1}{e^{-\left(s^{1 / v}+p\right) h}-1}+\frac{1}{1-e^{-\left(s^{1 / v}-p\right) h}}\right), L_{h, v}(\cosh p t)=\frac{h}{2}\left(\frac{1}{1-e^{-\left(s^{1 / v}+p\right) h}}+\frac{1}{1-e^{-\left(s^{1 / v}-p\right) h}}\right) .
$$

When $h \rightarrow 0$ and $v=1$, we get $L(\sinh p t)=\frac{p}{s^{2}-p^{2}}$ and $L(\cosh p t)=\frac{s}{s^{2}-p^{2}}$.

Proof. From (11), we have $L_{h, v}(\sinh p t)=(1 / 2) \Delta_{h}^{-1} e^{-s^{1 / v} t}\left(e^{p t}-e^{-p t}\right)$. Which completes the proof of (19). Similarly we can obtain $L_{h, v}(\cosh p t)$.

Theorem 14. If we denote $H_{r}=e^{-\left(s^{1 / v}+(n-2 r) a\right) h}-1, H_{-r}=e^{-\left(s^{1 / v}-(n-2 r) a\right) h}-1$, then

$$
\begin{gathered}
L_{h, v}\left(\sinh ^{n} p t\right)=\frac{h}{2^{n}} \sum_{r=0}^{[n / 2]}\left(\begin{array}{l}
n \\
r
\end{array}\right)\left(\frac{(-1)^{r}}{H_{r}}-\frac{(-1)^{r}}{H_{-r}}\right), \quad n \text { is odd. } \\
L_{h, v}\left(\sinh ^{n} p t\right)=\frac{h}{2^{n}} \sum_{r=0}^{[n / 2]-1}\left(\begin{array}{l}
n \\
r
\end{array}\right)\left(\frac{(-1)^{r+1}}{H_{r}}+\frac{(-1)^{r+1}}{H_{-r}}\right)+\left(\begin{array}{l}
n \\
\frac{n}{2}
\end{array}\right) \frac{2^{-n}(-1)^{r} h}{\left(1-e^{-s^{1 / v} h}\right)}, n \text { is even. } \\
L_{h, v}\left(\cosh ^{n} p t\right)=\frac{-h}{2^{n}} \sum_{r=0}^{[n / 2]}\left(\begin{array}{l}
n \\
r
\end{array}\right)\left(\frac{1}{H_{r}}+\frac{1}{H_{-r}}\right), \quad n \text { is odd. } \\
L_{h, v}\left(\cosh ^{n} p t\right)=\frac{-h}{2^{n}} \sum_{r=0}^{[n / 2]-1}\left(\begin{array}{l}
n \\
r
\end{array}\right)\left(\frac{1}{H_{r}}+\frac{1}{H_{-r}}\right)+\left(\begin{array}{l}
n \\
\frac{n}{2}
\end{array}\right) \frac{2^{-n} h}{\left(1-e^{-s^{1 / v} h}\right)}, \quad n \text { is even. }
\end{gathered}
$$

Proof. From $\sin h^{n} p t=\frac{1}{2^{n-1}} \sum_{r=0}^{[n / 2]}(-1)^{r}\left(\begin{array}{l}n \\ r\end{array}\right) \sin h(n-2 r) p t$ and (13), we get the proof of (20). Similarly, we can obtain the proof of (21), (22) and (23).

Theorem 15. Let $t \in(0, \infty), h>0$ and $s>0$, then $L_{h, v}\left(t_{h}^{(\mu)}\right)=\frac{h^{\mu+1} \mu ! e^{s^{1 / v} h}}{\left(e^{s^{1 / v} h}-1\right)^{\mu+1}}$.

Proof. Taking $u(t)=t_{h}^{(1)}$ in (11), we have $L_{h, v}\left(t_{h}^{(1)}\right)=\left.h \Delta_{h}^{-1} e^{-s^{1 / v}} t_{h}^{(1)}\right|_{t=0} ^{\infty}$. Now taking $u(t)=t_{h}^{(1)}, w(t)=$ $e^{-s^{1 / v} t}$ in (6) and applying (5), we get

$$
L_{h, v}\left(t_{h}^{(1)}\right)=h\left(\left.\frac{t_{h}^{(1)} e^{-s^{1 / v} t}}{\left(e^{-s^{1 / v} h}-1\right)}\right|_{t=0} ^{\infty}-\left.\frac{h e^{-s^{1 / v}}(t+h)}{\left(e^{-s^{1 / v} h}-1\right)^{2}}\right|_{t=0} ^{\infty}\right)=\frac{h^{2} e^{s^{1 / v} h}}{\left(e^{s^{1 / v} h}-1\right)^{2}}
$$

Now taking $u(t)=t_{h}^{(2)}$ in (11) and applying (6), and (5), we get $L_{h, v}\left(t_{h}^{(2)}\right)=\frac{h^{3} 2 ! e^{s^{1 / v} h}}{\left(e^{s^{1 / v} h}-1\right)^{3}}$. Repeating this process $n$ times, we get the proof of Theorem (15). 
Corollary 16. Let $t \in(0, \infty), h>0$ and $s>0$, then $L_{h, v}\left(t^{n}\right)=\sum_{r=0}^{n} \frac{S_{r}^{n} h^{n+1} n ! e^{s^{1 / v} h}}{\left(e^{s^{1 / v} h}-1\right)^{n+1}}$.

Proof. The proof follows from (ii) of (1), (ii) of (5) and Theorem (15).

Example 3. Taking $n=2$ in Theorem 15, we obtain

$$
L_{h, v}\left(t_{h}^{(2)}\right)=\frac{h^{3} 2 ! e^{s^{1 / v} h}}{\left(e^{s^{1 / v} h}-1\right)^{3}}=h \sum_{r=0}^{\infty}(r h)_{h}^{(2)} e^{-s^{1 / v} r h}
$$

which verified for the values $h=2, s=3$ and $v=0.7$ by MATLAB coding given below: $2 . * \operatorname{symsum}((r . * 2 . *$ $(r-1) . * 2) . * \exp (-3 . \wedge(1 . / 0.7) . * r . * 2), r, 0$, inf $)=(16 . * \exp (3 . \wedge(1 . / 0.7) . * 2)) . /((\exp (3 . \wedge(1 . / 0.7) . * 2)-$ 1). $\wedge 3)$.

Figure 1 is the input function(signal) as polynomial factorial for the time factor $t$ and Figure 2 is the fractional generalized Laplace transform in the frequency domain $s$ and also here in the frequency domain the fraction $v$ varies as $0.9,0.8,0.7,0.6,0.5$ which are generated by MATLAB.

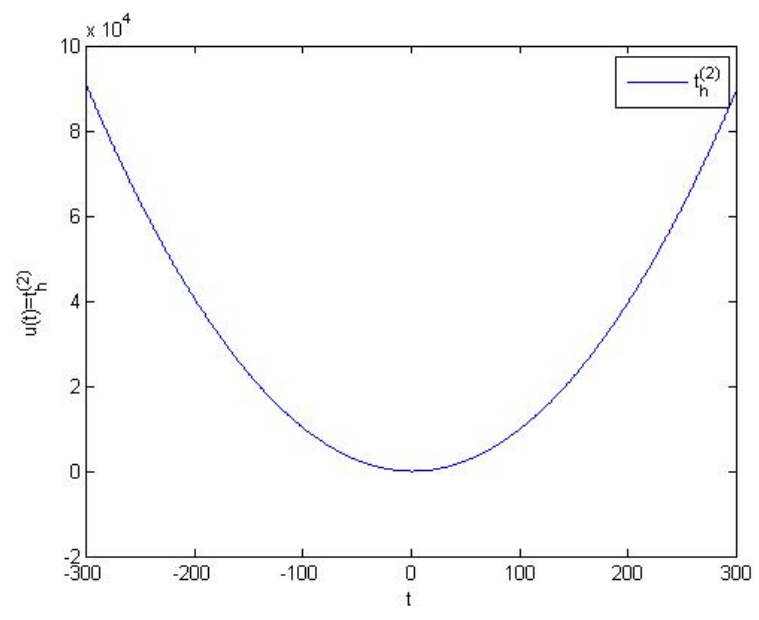

Figure 1. Time(t)

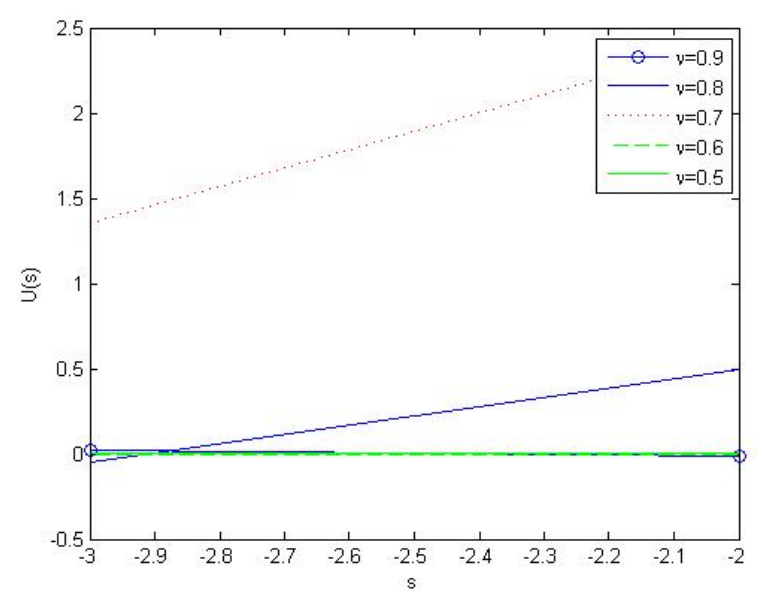

Figure 2. Frequency(s) 


\section{Convolution Product and Fractional Laplace Transforms}

In this section, we defined convolution product and its properties with Fractional Laplace transforms. The following definitions are motivated by [19] using difference operator.

Definition 17. Let $u(t)$ be the real valued function, then the incomplete generalized Laplace transform is defined by

$$
L_{h}[u(t), b]=\left.\Delta_{h}^{-1} u(t) e^{-s t}\right|_{0} ^{b}
$$

Definition 18. Let $u(t)$ and $v(t)$ are the two real valued functions, then the convolution product is defined by

$$
(u \circ v)(t)=\left.\Delta_{h}^{-1} u(\xi-t) v(\xi)\right|_{\xi=t^{\prime}} ^{\infty} \quad t>0 .
$$

The following lemma shows that the relation between convolution product and Fractional Laplace transform.

Lemma 19. Let $\mu \in R^{+}, u(t)$ and $v(t)$ are the real valued functions, then

1. $u \circ e^{-\mu^{1 / v}}=L_{h, v}[u] \cdot e^{-\mu^{1 / v}}$,

2. $L_{h, v}[u \circ v]=L_{h, v}\left[L_{h}\left(u\left(t_{1}\right), \xi\right)\right]$.

Proof. (1) From (27), we get $\left(u \circ e^{-\mu^{1 / v}}\right)(t)=\left.\Delta_{h}^{-1} u(\xi-t) e^{-\mu^{1 / v}}\right|_{\xi=t} ^{\infty}$. Taking $t_{1}=\xi-t$, which gives $\left(u \circ e^{-\mu^{1 / v}}\right)(t)=\left.\Delta_{h}^{-1} u\left(t_{1}\right) e^{-\mu^{1 / v}\left(t_{1}+t\right)}\right|_{t_{1}=0} ^{\infty}$. Then, $\left(u \circ e^{-\mu^{1 / v}}\right)(t)=\left.e^{-\mu^{1 / v}(t)} \Delta_{h}^{-1} u\left(t_{1}\right) e^{-\mu^{1 / v}\left(t_{1}\right)}\right|_{t_{1}=0^{\prime}} ^{\infty}$ which completes the proof of (1).

(2) Now $L_{h, v}[u \circ v](t)=\left.\Delta_{h}^{-1}(u \circ v)(t) e^{-\mu^{1 / v}(t)}\right|_{t=0} ^{\infty}=\left.\Delta_{h}^{-1}\left[\left.\Delta_{h}^{-1} u(\xi-t) v(\xi)\right|_{\xi=t} ^{\infty}\right] e^{-\mu^{1 / v}(t)}\right|_{t=0} ^{\infty}$. Now applying Fubini's Theorem, we get

$$
\left.\Delta_{h}^{-1} e^{-\mu^{1 / v}(t)}\left[\left.\Delta_{h}^{-1} u(\xi-t) v(\xi)\right|_{\xi=t} ^{\infty}\right]\right|_{t=0} ^{\infty}=\left.\Delta_{h}^{-1} v(\xi)\left[\left.\Delta_{h}^{-1} u(\xi-t) e^{-\mu^{1 / v}(t)}\right|_{t=0} ^{\xi}\right]\right|_{\xi=0} ^{\infty} .
$$

Then the proof of (2) follows by applying (26).

The following example illustrate the verification of convolution product.

Example 4. Consider the following functions

$$
u(t)=\left\{\begin{array}{cc}
e^{-s^{1 / v} t}, & t \in(0, \infty) \\
0, & \text { otherwise }
\end{array} \quad v(t)= \begin{cases}t, & t \in(0, \infty) \\
0, & \text { otherwise }\end{cases}\right.
$$

Now, from (27), we get $(u \circ v)(t)=\left.\Delta_{h}^{-1} e^{-s^{1 / v}(\xi-t)} \xi\right|_{\xi=0} ^{\infty}$. Then using (24), which gives $(u \circ v)(t)=$ $\frac{h^{2} e^{-s^{1 / v}}(h-t)}{\left(e^{-s^{1 / v} h}-1\right)^{2}}$. By (3), we get the relation as follows

$$
(u \circ v)(t)=h \sum_{r=0}^{\infty}(r h) e^{-s^{1 / v}(r h-t)}=\frac{h^{2} e^{-s^{1 / v}(h-t)}}{\left(e^{-s^{1 / v} h}-1\right)^{2}}
$$

which verified for the values $t=4, h=3, s=2$ and $v=0.3$ by MATLAB coding given below: $3 . *$ $\operatorname{symsum}(\exp (-2 . \wedge(1 . / 0.3) . *((r . * 3)-4)) . * r . * 3, r, 0$, inf $)=9 . * \exp (-2 . \wedge(1 . / 0.3) \cdot *(-1)) . /((\exp (-2 . \wedge$ $\left.(1 . / 0.3) \cdot * 3)-1)^{2}\right)$.

Figure 3 explains the input time(t) function $u(t)$ and $v(t)$ and Figure 4 tells that the convolution product of the functions in the frequency(s) domain as varying $v$ as $0.5,0.6,0.7$ which are generated by MATLAB. 


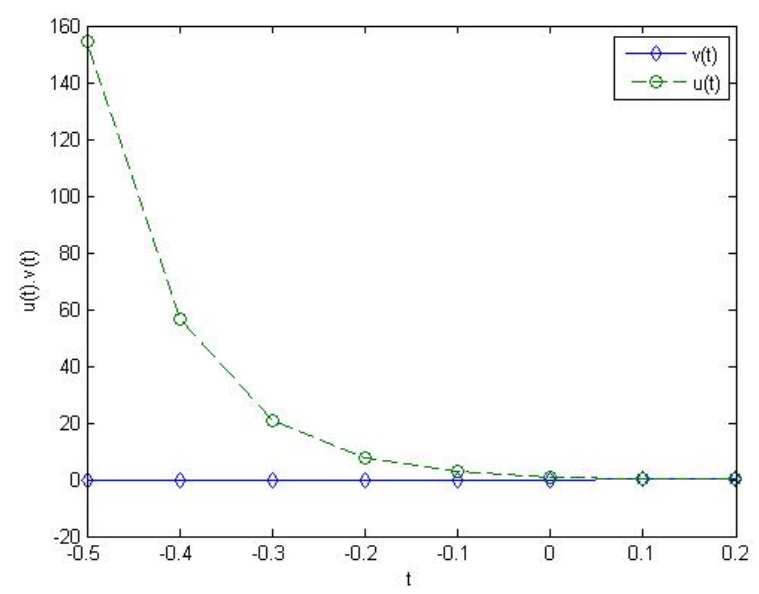

Figure 3. Time(t)

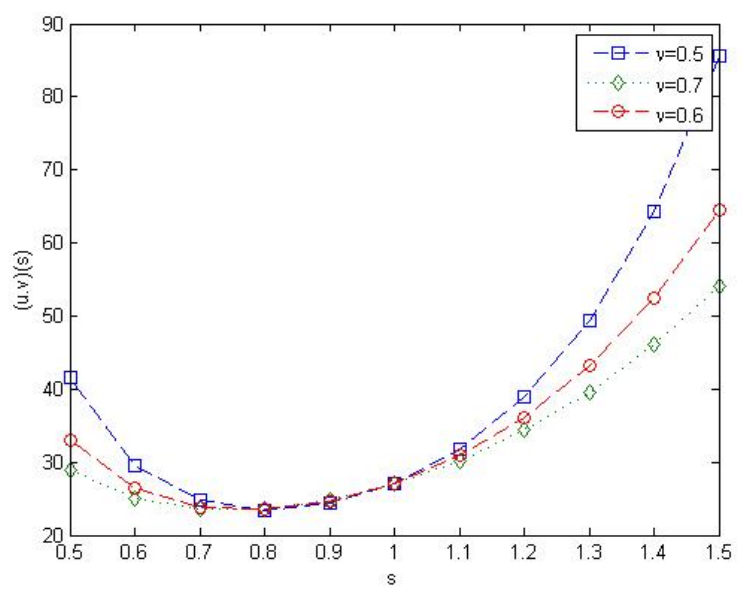

Figure 4. Frequency(s)

\section{Conclusion}

The fractional generalized Laplace transform is successfully defined and properties were presented. We derived formulaes and obtained its transform for certain functions like polynomial factorial, trigonometric functions, etc. When $v=1$ and $h \rightarrow 0$, we get classical Laplace transform. The convolution product defined and the relation between Laplace transform and convolution product were presented. We conclude this investigations and findings are verified and analyzed the outcomes in the time $(\mathrm{t})$ and frequency(s) domains with graphs.

Author Contributions: All authors contributed equally to the writing of this paper. All authors read and approved the final manuscript.

Conflicts of Interest: "The authors declare no conflict of interest."

\section{References}

[1] Miller, K. S., \& Ross, B. (1993). An introduction to the fractional calculus and fractional differential equations. John Wiley \& Sons, Inc., New York.

[2] Oldham, K., \& Spanier, J. (2002). The fractional calculus theory and applications of differentiation and integration to arbitrary order. Dover Publications, Inc., Mineola, New York.

[3] Podlubny, I. (1998). Fractional differential equations: an introduction to fractional derivatives, fractional differential equations, to methods of their solution and some of their applications (Vol. 198). Elsevier.

[4] Atici, F. M., \& Eloe, P. W. (2011). Two-point boundary value problems for finite fractional difference equations. Journal of Difference Equations and Applications, 17(04), 445-456. 
[5] Atici, F. M., \& Eloe, P. W. (2007). Fractional q-calculus on a time scale. Journal of Nonlinear Mathematical Physics, 14(3), 341-352.

[6] Atici, F. M., \& Eloe, P. W. (2007). A transform method in discrete fractional calculus. International Journal of Difference Equations, 2(2), 165-176.

[7] Goodrich, C. S. (2010). Solutions to a discrete right-focal fractional boundary value problem. International Journal of Difference Equations, 5(2), 195-216.

[8] Goodrich, C. S. (2010). Continuity of solutions to discrete fractional initial value problems. Computers $\mathcal{E}$ Mathematics with Applications, 59(11), 3489-3499.

[9] Goodrich, C. S. (2011). Some new existence results for fractional difference equations. International Journal of Dynamical Systems and Differential Equations, 3(1-2), 145-162.

[10] Holm, M. (2011). Sum and difference compositions in discrete fractional calculus. Cubo (Temuco), 13(3), $153-184$.

[11] Smith, S. W. (1997). The scientist and engineer's guide to digital signal processing(Second Edition). California Technical Publishing San Diego, California.

[12] Bohner, M., \& Peterson, A. (2012). Dynamic equations on time scales: An introduction with applications. Springer Science \& Business Media.

[13] Ivic, A. (2015). Some applications of Laplace transforms in analytic number theory. Novi Sad Journal of Mathematics, $45(1), 31-44$.

[14] Ivic, A. (2000). The Laplace transform of the fourth moment of the zeta-function. Publikacije Elektrotehnickog fakulteta. Serija Matematika, 41-48.

[15] Sedletskii, A. M. (2014). Fourier transforms and approximations. CRC press.

[16] Jutila, M. (2001). The Mellin transform of the square of Riemann's zeta-function. Periodica Mathematica Hungarica, 42(1-2), 179-190.

[17] G. Britto Antony Xavier, B. Govindan, S. John Borg and M. Meganathan, Generalized Laplace Transform arrived from an Inverse Difference Operator, Global Journal of Pure and Applied Mathematics, 12(3) (2016),661-666.

[18] Britanak, V., \& Rao, K. R. (1999). The fast generalized discrete Fourier transforms: A unified approach to the discrete sinusoidal transforms computation. Signal Processing, 79(2), 135-150.

[19] Medina, G. D., Ojeda, N. R., Pereira, J. H., \& Romero, L. G. (2017). Fractional Laplace Transform and Fractional Calculus. International Mathematical Forum, 12(20), 991-1000.

(C) 2019 by the authors; licensee PSRP, Lahore, Pakistan. This article is an open access article distributed under the terms and conditions of the Creative Commons Attribution (CC-BY) license (http://creativecommons.org/licenses/by/4.0/). 